\title{
Vedolizumab for perianal fistulizing Crohn's disease: systematic review and meta-analysis
}

\author{
Fares Ayoub, Matthew Odenwald, Dejan Micic, Sushila R. Dalal, Joel Pekow, Russell D. Cohen, David T. Rubin, \\ Atsushi Sakuraba \\ Inflammatory Bowel Disease Center, Section of Gastroenterology, Hepatology, and Nutrition, University of Chicago, Chicago, IL, USA
}

Background/Aims: Perianal fistulas are a debilitating manifestation of Crohn's disease (CD). Despite the advent of anti-tumor necrosis factor (anti-TNF) therapy, the medical management of fistulizing CD continues to be challenged by unmet needs. We conducted a systematic review and meta-analysis of the effectiveness of vedolizumab for the management of perianal fistulizing CD. Methods: A search of PubMed, EMBASE and the Cochrane Library was performed from inception to June 2020 for studies reporting rates of perianal fistula healing in CD patients treated with vedolizumab. The primary outcome of interest was complete healing of perianal fistulas and the secondary outcome was partial healing. The pooled fistula healing rates with 95\% confidence intervals (CI) were calculated utilizing a random effects model. Results: A total of 74 studies were initially identified, 4 of which met the inclusion criteria. A total of 198 patients with active perianal fistulas were included, $87 \%$ of whom had failed previous anti-TNF therapy. The pooled complete healing rate was $27.6 \%$ (95\% CI, $18.9 \%-37.3 \%$ ) with moderate heterogeneity $\left(I^{2}=49.4 \%\right)$ and the pooled partial healing rate was $34.9 \%(95 \% \mathrm{CI}, 23.2 \%-47.7 \%)$ with high heterogeneity $\left(I^{2}=67.1 \%\right)$. Conclusions: In a meta-analysis of 4 studies that included 198 patients with perianal fistulizing CD, the majority of whom had failed previous anti-TNF therapy, vedolizumab treatment led to healing of perianal fistulas in nearly one-third of the patients. The lack of high-quality data and significant study heterogeneity underscores the need for future prospective studies of fistula healing in patients receiving anti-integrin therapy. (Intest Res 2022;20:240-250)

Key Words: Vedolizumab; Perianal fistula; Perianal disease; Crohn disease; Meta-analysis

\section{INTRODUCTION}

Crohn's disease (CD) is a lifelong inflammatory bowel disease of unknown etiology. CD may involve any part of the gastrointestinal tract and can manifest in distinct clinicopathologic phenotypes including a stricturing, penetrating (fistulizing), or inflammatory (non-stricturing, non-penetrating) phenotype. An estimated $20 \%$ to $40 \%$ of CD patients develop a fistula in their lifetime $\mathrm{e}^{1,2}$ and at least 77,000 patients are estimated to

Received June 28, 2021. Revised September 8, 2021.

Accepted September 22, 2021.

Correspondence to Atsushi Sakuraba, Inflammatory Bowel Disease Center, Section of Gastroenterology, Hepatology, and Nutrition, University of Chicago, 5841 S. Maryland Avenue, MC 4076, Chicago, IL 60637, USA. Tel: +1-773-834-1345, Fax: +1-773-702-9399, E-mail: asakurab@medicine. bsd.uchicago.edu live with fistulizing CD in the United States. ${ }^{3}$ Given the significant morbidity associated with fistulizing disease, this is a substantial complication of CD.

Perianal fistulas are the most common manifestation of fistulizing $\mathrm{CD}$ and adequate management frequently requires a combined surgical and medical approach. Anti-tumor necrosis factor $\alpha$ (anti-TNF- $\alpha$ ) biologics, particularly infliximab, are widely used for perianal fistulizing $\mathrm{CD}$. In the pivotal placebocontrolled randomized trial of infliximab for fistulizing CD, complete fistula remission within 18 weeks was observed in $13 \%$ of patients receiving placebo, $55 \%$ of patients receiving 5 $\mathrm{mg} / \mathrm{kg}$ infliximab every 8 weeks $(P=0.001)$, and $38 \%$ of patients receiving $10 \mathrm{mg} / \mathrm{kg}$ every 8 weeks $(P=0.04) .{ }^{4}$ Similar healing rates were seen in a post-hoc analysis of the pivotal maintenance trial of adalimumab, and both anti-TNF thera- 
pies can be augmented with ciprofloxacin. ${ }^{5-7}$ However, there is a paucity of randomized, controlled trials evaluating the efficacy of non-TNF biologics for perianal fistulizing disease.

Vedolizumab is a gut-selective $\alpha_{4} \beta_{7}$ integrin antibody that is effective for induction and maintenance of remission of $\mathrm{CD}^{8}$ It has been shown to be effective in $\mathrm{CD}$ patients who are antiTNF naïve, in those who are primary nonresponders to antiTNFs, and those who develop secondary nonresponse due to mechanistic escape, development of antibodies or drug-related adverse events. ${ }^{9}$ There remains uncertainty about the effectiveness of vedolizumab for the management of perianal fistulizing CD. We therefore conducted a systematic review and meta-analysis of the evidence of the effectiveness of vedolizumab for perianal fistulizing CD.

\section{METHODS}

This meta-analysis was performed in accordance with the criteria established in the Preferred Reporting Items for Systematic Reviews and Meta-Analyses (PRISMA) and Meta-Analysis of Observational Studies in Epidemiology (MOOSE) guidelines. This study was registered with the University of York International Prospective Register Of Systematic Reviews (PROSPERO; Registration number CRD42020209930). ${ }^{10}$

\section{Search Strategy and Study Selection}

Studies were identified by performing a literature search of 3 electronic databases (MEDLINE through PubMed, EMBASE and the Cochrane Central Register of Controlled Trials) with the last search performed in June 2020. The detailed search strategy is outlined in Supplementary Table 1. We attempted to identify additional studies by reviewing the reference list of all included studies and performing a manual search to retrieve other relevant articles that may have been missed with the initial search strategy. There was no language restriction and manuscripts were translated to English if necessary. Authors of manuscripts were contacted when any data were missing in the manuscripts. Two investigators (F.A. and M.O.) screened all titles and abstracts for relevance to the study. The full text of potentially eligible studies was subsequently reviewed by 2 investigators (F.A. and M.O). Disagreements were resolved by consensus or by consulting with a third investigator (A.S.).

\section{Inclusion and Exclusion Criteria}

Inclusion criteria were: (1) retrospective or prospective, case series (larger than 10 patients), case-control, or cohort studies and clinical trials (including randomized clinical trials); (2) studies including adult patients with CD treated with vedolizumab; (3) studies reporting number of patients with perianal fistulizing disease; and (4) studies reporting fistula-specific outcomes of patients treated with vedolizumab. Exclusion criteria were: (1) studies not reporting numbers of included patients with perianal fistulizing disease; (2) studies not reporting fistula-specific outcomes; (3) reviews, commentaries, surveys; (4) and duplicate studies.

\section{Data Extraction}

Data from each eligible study were extracted using a standardized data extraction sheet. The extracted data included: (1) study authors, (2) year of publication, (3) setting (location), (4) study type (retrospective or prospective/single-center or multicenter), (5) patient demographics (age, sex), (6) number of patients with active perianal disease at initiation of vedolizumab, (7) number of patients with setons at initiation of vedolizumab, (8) previously failed CD therapies, (9) vedolizumab dosage and frequency, (10) number of patients who had vedolizumab dose escalation, (11) number of patients on concomitant immunomodulator, antibiotic or steroid therapy, (12) definitions of active perianal fistula and fistula healing, (13) time point for assessment of healing, (14) number of patients with partial healing, (15) number of patients with complete healing, and (16) number of patients with recurrent or de novo fistulas during treatment. Due to the nature of this research article not involving individual patients or patient data (meta-analysis), ethical approval was not required or obtained from the University of Chicago.

\section{Outcomes and Definitions}

The primary aim of this study was to conduct a meta-analysis assessing the rates of complete healing of perianal fistulizing disease in CD patients treated with vedolizumab. A secondary aim was to assess the rates of partial healing of perianal fistulizing disease. Active perianal fistulizing disease was defined by clinical and/or imaging evidence including perianal abscess, perianal external openings with drainage, and/or imaging evidence demonstrating perianal abscess with or without fistula tract formation. Complete healing was defined by investigatordetermined complete resolution of perianal pain, fluctuation or drainage with/without imaging evidence in support of healing, without the need for any additional medical and/or surgical therapy directed at the perianal disease. ${ }^{11}$ Partial healing was defined as at least $50 \%$ reduction from baseline in the 
number of draining perianal fistulas (of those draining at baseline). ${ }^{12}$ We also collected data on the rates of recurrent or $d e$ novo fistula formation during treatment in patients with inactive perianal disease or no history of perianal disease.

\section{Assessment of Methodologic Quality}

The quality of studies was assessed using the Newcastle-Ottawa Scale (NOS) ${ }^{13}$ Since the majority of included studies were not randomized placebo-controlled trials, we utilized a modified version of the NOS appropriate for our analysis. This tool removes from the NOS the items that relate to comparability between 2 arms and retains items that assess representation and selection of cases as well as ascertainment of exposure and outcome. ${ }^{13}$ A point is assigned to each component of the modified scale, with the highest possible score being $6 / 6$. Studies were considered to be high quality if they scored 6/6, moderate quality if they scored $5 / 6$ and low quality if they scored $4 / 6$ or less. The quality of all studies was assessed by 2 investigators (F.A. and M.O.). Since less than 10 studies were included, visual inspection of the funnel plot was used to assess for publication bias. ${ }^{14}$

\section{Statistical Analysis}

The pooled rates were calculated utilizing a random effects model and the Freeman-Tukey arcsine transformation was used. ${ }^{15}$ The Cochran Q test and $I^{2}$ were used to assess heterogeneity of included studies. $I^{2}$ values of $<25 \%, 25 \%-50 \%$, and $>50 \%$ were considered to represent low, moderate, and high heterogeneity, respectively. ${ }^{14} P$-values $<0.05$ were considered significant and all tests were two-tailed. The study was performed in accordance with the PRISMA recommendations for reporting systematic reviews and meta-analyses. Analysis was conducted using Stata, version 15 (StataCorp., College Station, TX, USA).

\section{RESULTS}

\section{Search Results}

The flow diagram for study selection is depicted in Fig. 1. Overall, 72 studies were identified using our search strategy from database search and 2 additional records were identified through other sources. Eleven items were duplicates and were excluded. Of the remaining 63 studies after duplicate removal, 53 were excluded after screening titles and abstracts. Full text review was then performed on 10 studies using the predefined inclusion and exclusion criteria, after which 4 studies ${ }^{11,12,16,17}$ were retained. Two of the 4 studies were randomized, controlled clinical trials (one was a post-hoc exploratory analysis of the pivotal GEMINI 2 randomized, placebo-controlled vedolizumab maintenance trial, ${ }^{16}$ the other was a randomized trial of 2 vedolizumab dosing regimens; standard dosing versus standard dosing plus an additional dose at week $10^{12,18}$ ). The other 2 studies were retrospective cohort studies. ${ }^{11,17}$ Of
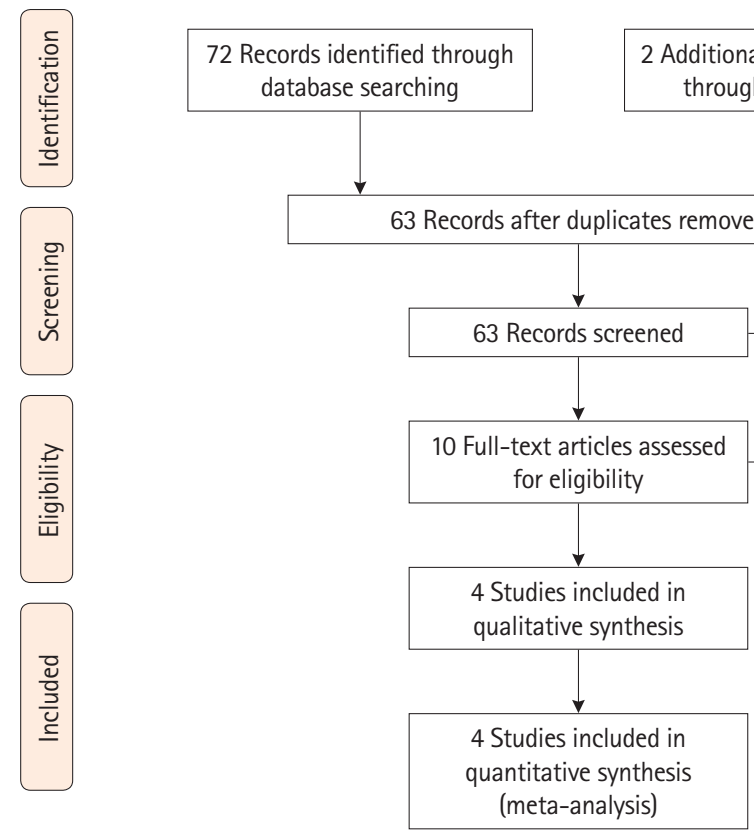

Additional records identified through other sources

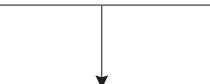

Fig. 1. PRISMA (Preferred Reporting Items for Systematic Reviews and Meta-Analyses) flow diagram depicting the selection process of studies used in the analysis. 
note, all patients in the study by Feagan et al. ${ }^{16}$ received vedolizumab induction therapy, but were then randomized to maintenance with either placebo or vedolizumab. Only outcomes of those who received both vedolizumab induction and maintenance therapy were included in our analysis to allow for appropriate comparisons with the other included studies.

All studies were published between 2018 and 2020. All were multicenter studies, 2 of which were conducted internationally and 2 in France. Of note, the study by Pestour et al. was presented at the 13th Congress of the European Crohn's and Colitis Organisation (ECCO) and published in abstract form ${ }^{19}$ and also published as a peer-reviewed Master's degree thesis document in an open-access web repository utilized by French higher-education institutions (Larchive ouverte pluridisciplinaire [HAL]). ${ }^{17}$ The study by Schwartz et al. was presented at Digestive Disease Week (DDW) 2020 and published in abstract form ${ }^{18}$ with the final full results of the clinical trial posted publicly on the European Union Clinical Trials Register; this final version was used for this analysis. ${ }^{12}$

\section{Patient Population and Study Characteristics}

A total of 198 patients with active perianal fistulizing disease were treated with vedolizumab induction and maintenance. Study and patient characteristics are summarized in Table 1. All studies except the study by Pestour et al. ${ }^{17}$ reported age and sex of included patients; mean age ranged from 32.6 to 35.1 years and 74 out of 169 patients (44\%) were males. The modality for assessment of fistula healing was reported by all studies except the study by Pestour et al. ${ }^{17}$ the remainder of studies used clinical examination to assess for healing, with 2 studies ${ }^{11,12}$ using imaging in addition to examination. The clinical trial by Schwartz et al. ${ }^{12}$ utilized the most comprehensive assessment for fistula healing, combining clinical examination, magnetic resonance imaging as well as several validated indices for perianal disease activity. Studies varied in their time points for primary outcome assessment from as short as 24 weeks following induction with vedolizumab ${ }^{17}$ up to 52 weeks. ${ }^{11,16}$ Only 1 study ${ }^{11}$ reported the number of patients with setons at the time of induction where 61 out of 102 patients $(60 \%)$ had setons and only 9 out of 61 (15\%) had successful seton removal during follow-up. Two studies ${ }^{11,16}$ reported need for surgical intervention directed at the perianal disease during vedolizumab therapy, both considered need for surgery a failure of vedolizumab. In the study by Chapuis-Biron et al., incision and drainage were required in 21 patients and 9 patients required fecal diversion. In the trial by Feagan et al., 3 patients had "fis- tula surgery" (1 during induction and 2 during maintenance), however the type of surgery was not specified.

All studies reported previous biologic therapies for patients, with the 2 studies $^{11,17}$ where all patients had failed prior antiTNF therapy, one study ${ }^{16}$ where 19 out of 39 (49\%) and anoth$\mathrm{er}^{12}$ where 22 out of 28 (79\%) patients had failed prior antiTNF therapy. Overall, $87 \%$ of patients had failed previous antiTNF therapy. Reasons for previous failure of anti-TNF therapy were not described in any of the studies.

\section{Treatment Characteristics}

All studies reported their vedolizumab dosing regimens (Table 2). All utilized standard dosing with vedolizumab $300 \mathrm{mg}$ administered intravenously at weeks $0,2,6$ (induction) and then every 4 or 8 weeks thereafter (maintenance) and the randomized clinical trial by Schwartz et al. ${ }^{12}$ compared this standard regimen to a similar regimen plus an additional dose at week 10. The 2 studies included randomized clinical trials ${ }^{12,16}$ did not allow dose escalation if no clinical response was noted, with the other 2 retrospective studies ${ }^{11,17}$ allowing dose escalation and reported escalation dosing regimens. In the study by Chapuis-Biron, 62 out of 102 patients (61\%) required dose escalation and the study by Pestour et al. did not report the number of patients requiring dose escalation. Three studies ${ }^{11,12,16}$ reported the number of patients on concomitant immunomodulator therapy with overall 66 out of 169 patients (39\%) on a stable dose of concomitant therapy with an immunomodulator. Two studies reported the number of patients who required any antibiotic therapy throughout the study with 60 out of 141 (43\%) patients, however, no details were provided on dosing, type, or duration of antibiotic therapy. Two studies $^{12,16}$ reported that 28 out of 67 (42\%) patients required concomitant steroid therapy throughout the study, but no details were provided on dosing or duration of therapy.

\section{Severity of Perianal Disease}

Two of four studies provided descriptive information on the severity and number of perianal fistulas, however these data were not uniform. Feagan et al. reported the number of draining fistulas per patient with 13 patients having 1 draining fistula, 4 with 2 draining fistulas and 1 with 3 or more draining fistulas. Pestour et al. described the severity of perianal disease, with isolated fistulas in most cases $(n=18 ; 67 \%)$, fistulas associated with anal stenosis $(\mathrm{n}=4 ; 15 \%)$, fistulas associated with anal ulcers $(\mathrm{n}=3 ; 11 \%)$, and fistulas associated with both anal stenosis and anal ulcer $(\mathrm{n}=2 ; 7 \%)$. 


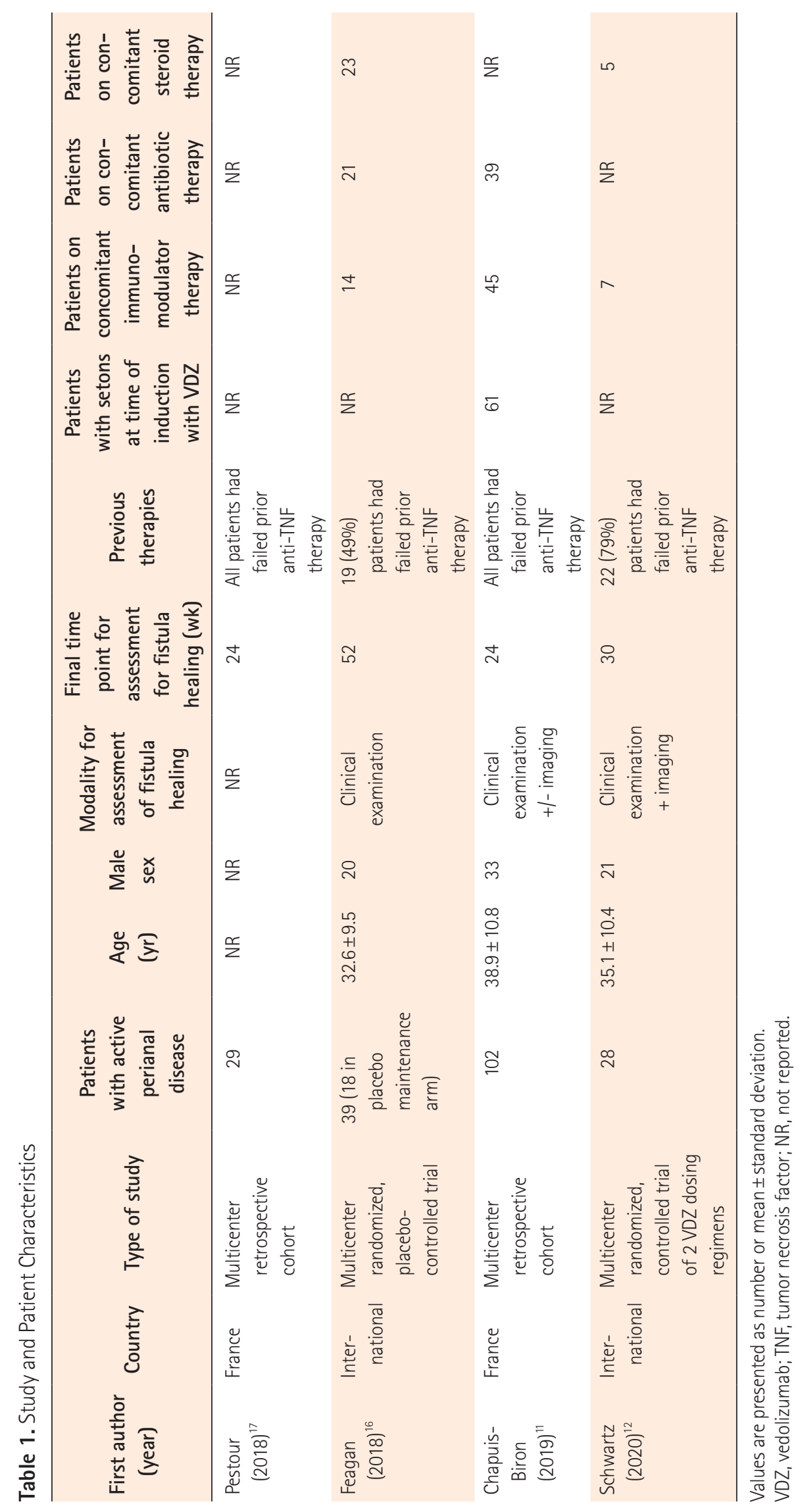




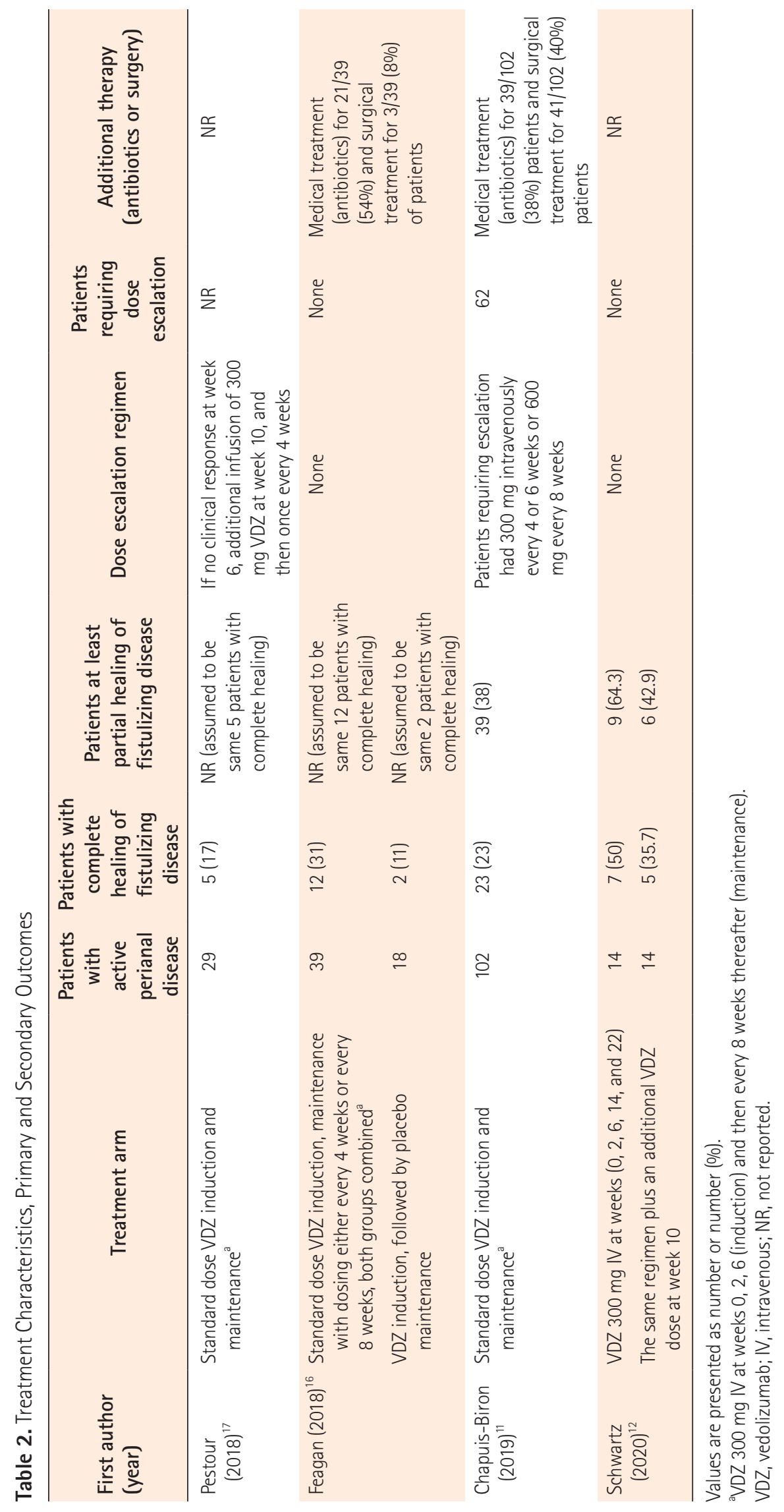




\section{Primary and Secondary Outcomes: Complete and Partial Fistula Healing}

All studies reported the primary outcome, rate of complete healing of perianal fistulizing disease (Fig. 2). In the randomized trial by Schwartz et al., ${ }^{12}$ of the 28 initially enrolled patients with perianal disease, 8 had incomplete data at the time of primary outcome assessment, and those were counted as nonresponders in an intention-to-treat analysis.

Overall, 52 out of 198 patients had complete healing of perianal fistulizing disease, with a pooled complete healing rate of $27.6 \%$ (95\% confidence interval [CI], 18.9\%-37.3\%) and moderate heterogeneity identified in the pooled analysis $\left(I^{2}=49.4 \%\right)$. Two of 4 studies ${ }^{11,12}$ explicitly reported the number of patients with partial healing (rather than complete healing) of perianal fistulizing disease (Fig. 3), with the 2 remaining studies only reporting rates of complete healing. A total of 71 out of 198 patients had at least partial healing of their perianal fistulizing disease, with a pooled partial healing rate of $34.9 \%$ (95\% CI, 23.2\%-47.7\%) and high heterogeneity identified in the pooled analysis $\left(I^{2}=67.1 \%\right)$. The noted moderate to significant heterogeneity in both pooled estimates can likely be explained by differences in sample size, in the rates of previous anti-TNF failure, rates of concomitant steroid or immunomodulator use, lack of detailed information on seton use in all studies, and the variable severity of perianal disease in the studied cohorts. Sufficient patient data were not available to perform an analysis focused on patients who had failed anti-TNF compared to those who were biologic-naïve. There was no evidence of substantial

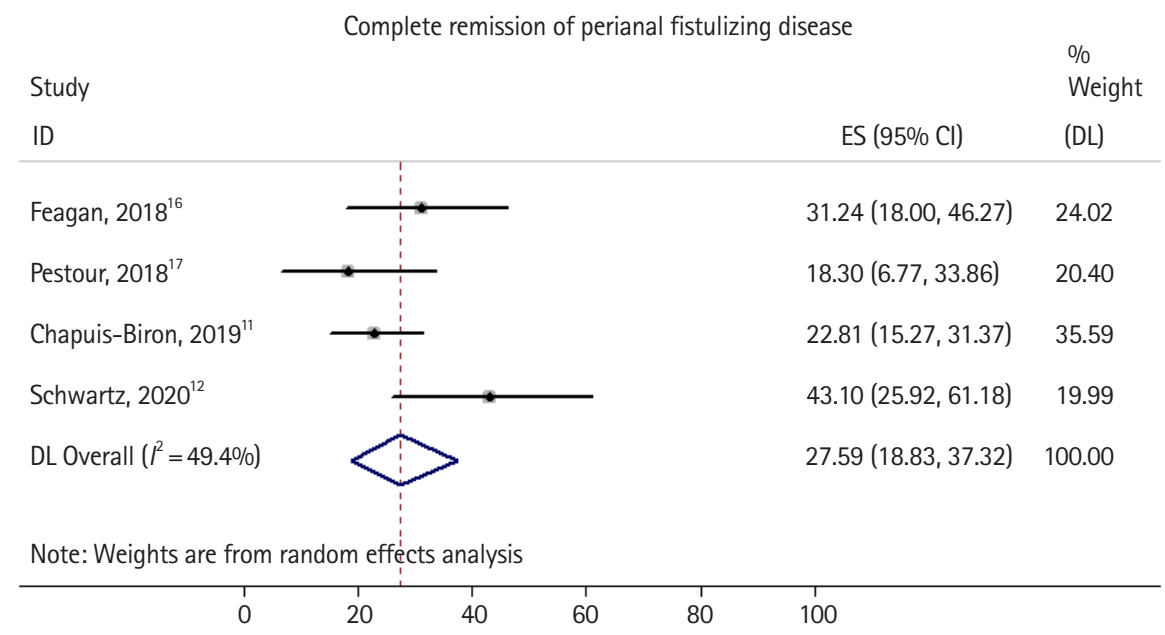

Fig. 2. Forrest plot demonstrating pooled rate and $95 \%$ confidence interval $(\mathrm{Cl})$ of complete healing of perianal fistulizing disease. ES, effect size; DL, DerSimonian-Laird method.

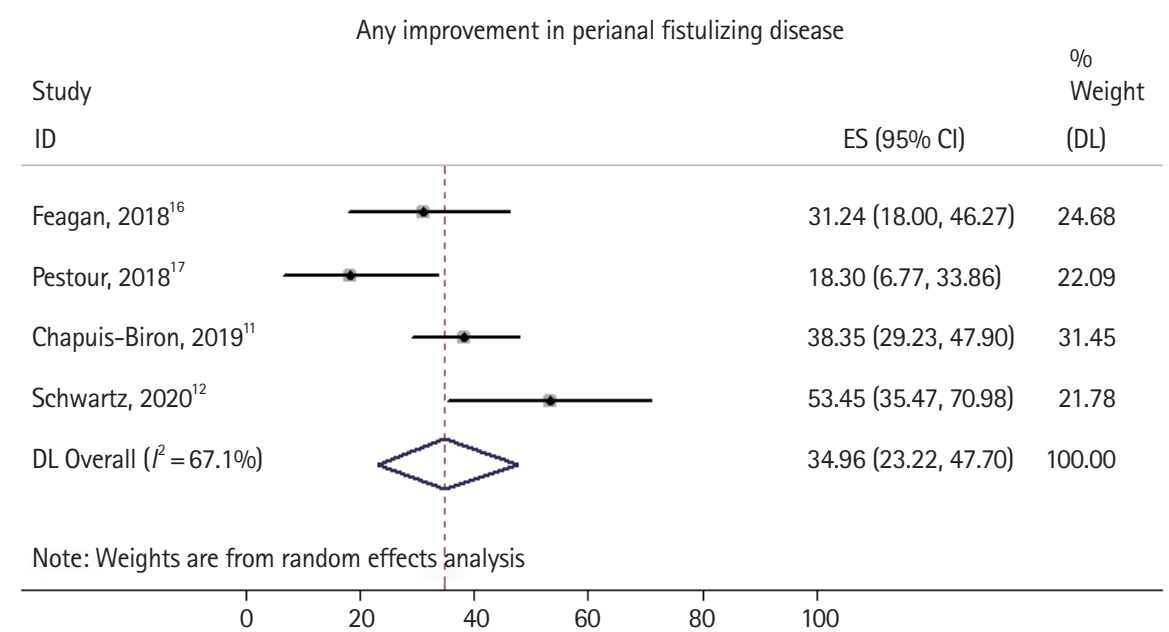

Fig. 3. Forrest plot demonstrating pooled rate and 95\% confidence interval (CI) of partial healing of perianal fistulizing disease. ES, effect size; DL, DerSimonian-Laird method. 
publication bias based on visual inspection of the funnel plot (Supplementary Fig. 1).

\section{Recurrence (or De Novo Incidence) of Perianal Disease in Patients with Inactive (or No) Disease at Baseline}

Two studies ${ }^{11,16}$ reported recurrence or de novo incidence of perianal disease in patients with inactive or no perianal disease at baseline. The randomized, controlled study by Schwartz only enrolled patients with active perianal disease and this outcome was not applicable. In a total of 357 patients, $23(6 \%)$ had either de novo or recurrent perianal fistulizing disease during induction and maintenance treatment with vedolizumab. In the retrospective study by Chapuis-Biron et al., 15 out of 49 patients with inactive perianal disease had a recurrence and in the randomized trial by Feagan et al., 8 patients had de novo perianal disease while on vedolizumab.

\section{Quality Assessment}

The risk of bias in the 4 studies was evaluated according to the modified Newcastle-Ottawa assessment scale and is shown in Supplementary Table 2. Overall, 2 of the 4 studies were found to be of high quality, 1 moderate quality, and 1 was found to be low quality.

\section{DISCUSSION}

In our meta-analysis of 198 patients with perianal fistulizing $\mathrm{CD}$, induction and maintenance therapy with vedolizumab led to complete healing of perianal fistulizing disease in $27.6 \%$ of patients and at least partial healing in $34.9 \%$. Notably, the majority (87\%) of included patients had failed prior anti-TNF therapy suggesting an already challenging disease phenotype. Nonetheless, our findings suggest vedolizumab may have numerically comparable perianal fistula healing rates to anti-TNF agents. ${ }^{20-22}$

Our meta-analysis adds to the limited literature available for clinicians faced with management of refractory perianal fistulizing CD. The majority of included subjects in our analysis had failed previous anti-TNF therapy. The causes of failure (primary vs. secondary nonresponse, adverse events) were not adequately described in the included studies. Two of our studies were from France, where vedolizumab is only reimbursed after anti-TNF failure, ${ }^{11}$ explaining the predominance of patients who had failed anti-TNF therapy in those studies. In 1 included study, ${ }^{11}$ at least $81 \%$ of patients had failed 2 or more anti-TNF agents. Overall, these factors may suggest a more resistant disease phenotype in our analyzed cohort; such that vedolizumab may possibly have an even higher success rate in biologic-naïve patients as has been previously demonstrated in other studies of vedolizumab. ${ }^{23}$ It is important to interpret our pooled results while considering the heterogeneity of the underlying study subjects. Several factors are known to modulate fistula healing including concurrent seton use, antibiotics, steroids and/or immunomodulators. Granular data were not available to perform subgroup analyses factoring in these different variables. For example, the study by ChapuisBiron et al. ${ }^{11}$ indicated antibiotic use was associated with a lower rate fistula healing, perhaps due to a more severe phenotype in that subgroup of patients.

In practice, vedolizumab is mostly used as second- or thirdline therapy in fistulizing CD due to both payor issues and the lack of robust effectiveness data as compared to anti-TNF agents. ${ }^{24}$ An examination of the mechanism of action for vedolizumab and TNF inhibitors, as well as the pathophysiology of fistulizing disease offers insights into their differential effectiveness in fistulizing disease. Vedolizumab is a monoclonal antibody against integrin $\alpha_{4} \beta_{7}$ which is expressed on a subset of T-cells. In vivo, vedolizumab blocks the interaction between the $\alpha_{4} \beta_{7}$ integrin and mucosal addressin cell adhesion molecule-1, which is mainly expressed on gastrointestinal tract endothelial cells. ${ }^{25}$ This mechanism of action allows vedolizumab to be "gut-selective," primarily reducing migration of T-lymphocytes into the gastrointestinal tract. In comparison, TNF inhibitors neutralize the activity of TNF- $\alpha$ in both its soluble and transmembrane forms across various tissues with downstream effects on several inflammatory pathways. ${ }^{26}$

While inflammation may allow the initial epithelial defect for required for fistula formation, epithelial to mesenchymal transition (EMT) of intestinal epithelial cells leads to downregulation of cell adhesion molecules, such as epithelial cadherin, loss of epithelial cell polarity, and migration of mesenchymal-like cells into the deeper layers to form fistula tracts. ${ }^{27,28}$ Multiple cytokines coordinate to promote the EMT to allow for deeper penetration of the migrating cells: TNF- $\alpha$ produced by inflammatory cells; transforming growth factor $\beta$ and interleukin-13 produced by fibroblasts; and matrix metalloproteinases that degrade the extracellular matrix. ${ }^{29}$ There is marked upregulation of TNF and the TNF receptor on transitional (mesenchymal-like) cells within perianal fistula tracts. ${ }^{30}$ Given that TNF- $\alpha$ is a central regulator of both the inflammation that results in an initial epithelial defect and the EMT, it is not sur- 
prising that TNF neutralizing antibodies are effective in treating penetrating $\mathrm{CD}$. The potential mechanism of more selective therapies, such as vedolizumab, in perianal $\mathrm{CD}$ is more recently being elucidated. A recent study of 7 patients with perianal fistulae found a significant number of T-cells in curettage material from the fistula tracts, and of the total $\mathrm{CD}^{+} \mathrm{T}$-cells, $68.7 \%$ were $\mathrm{CD3}^{+} \alpha_{4} \beta_{7}{ }^{+}$. While not establishing a causal relationship, the presence of $\alpha_{4} \beta_{7}^{+}$T-cells within fistula tracts lends some mechanistic support to the exploration of vedolizumab as a therapy for perianal fistulizing $\mathrm{CD}{ }^{31}$

Our analysis has several limitations, largely owing to the quality and scarcity of data on vedolizumab in perianal fistulizing $\mathrm{CD}$. The limited number of included studies and the underlying heterogeneity of study subjects may limit the generalizability of our results to the broader fistulizing $\mathrm{CD}$ population, particularly those with enteric and other non-perianal fistulas. While 2 of the included 4 studies were prospective randomized clinical trials, only 1 was a placebo-controlled trial but only for the maintenance phase of therapy, limiting our ability to generate odds ratios for fistula healing as compared to placebo. While direct comparisons to other therapies cannot be made due to different underlying patient populations, the numerical rates of response found in our study were comparable to those seen with anti-TNF agents, ${ }^{22}$ in an arguably more challenging disease phenotype since the majority of patients in our analysis had failed anti-TNF agents. To highlight these similarities, we extracted data on anti-TNF therapy for induction of healing of fistulizing $\mathrm{CD}$ as reported in a robust meta-analysis by Lee et al. ${ }^{22}$ from a total of 6 controlled studies. ${ }^{4,20,32-35}$ We used a similar statistical approach and found that anti-TNF therapy led to induction of complete healing of fistulizing $\mathrm{CD}$ in a total of 92 out of 267 patients, with a pooled rate of $29.7 \%$ ( $95 \%$ CI, $18.3 \%-42.6 \%)$. Finally, there was some variability in the time points and the modalities used for assessment of the primary outcome of fistula healing, widening the true $\mathrm{CI}$ of our pooled estimates.

A future randomized, placebo-controlled trial including biologic naïve patients with balanced underlying baseline characteristics and concurrent medical and surgical treatment characteristics would allow insights into the true efficacy of vedolizumab for fistulizing disease. However, the realities of CD clinical trial logistics and enrollment make the likelihood of such a trial being conducted quite low. This is exemplified by the difficulties faced by Schwartz et al. ${ }^{12}$ in their well-designed international prospective, randomized, controlled trial for perianal fistulizing CD comparing standard vedolizumab dosing ver- sus standard dosing plus an additional dose at week 10 (ENTERPRISE) which closed enrollment early after enrolling 34 subjects, only 20 of which ultimately had complete data for the final analysis. These limitations are increasingly recognized by the wider IBD research community, with a recent analysis finding that the average recruitment rate in moderate-to-severe CD decreased from 0.65 to 0.10 patients per site per month in the 20 years from 1998 to $2018 .^{36}$

The management of perianal fistulizing CD continues to be challenging for both patients and physicians. While anti-TNF agents have demonstrated efficacy in induction of remission, long-term healing is achieved only in $50 \%$ of patients. ${ }^{37}$ In this meta-analysis of 4 studies including 198 patients with perianal fistulizing $\mathrm{CD}$, the majority of which had failed previous antiTNF therapy, vedolizumab led to complete healing of perianal fistulizing disease in $27.6 \%$ of patients and at least partial healing in 34.9\%. Future studies designed to control for concurrent surgical and medical therapies and enrolling a larger number of biologic naïve patients are warranted.

\section{ADDITIONAL INFORMATION}

\section{Funding Source}

The authors received no financial support for the research, authorship, and/or publication of this article.

\section{Conflict of Interest}

This was an investigator-initiated study. There was no involvement of the drug manufacturer in either the conception, data collection, analysis, or manuscript writing. Rubin DT is a consultant to AbbVie, AbGenomics, Allergan Inc., Amgen, Celgene Corporation, Forward Pharma, Genentech/Roche, Janssen Pharmaceuticals, Merck \& Co Inc., Miraca Life Sciences, Mitsubishi Tanabe Pharma Development America Inc., Napo Pharmaceuticals, Shire, Takeda, and Target Pharma Solutions and has received grant support from AbbVie, Genentech/Roche, Janssen Pharmaceuticals, Prometheus Laboratories, Shire, Takeda, and UCB Pharma. Cohen RD serves on the speaker's bureau for AbbVie and Takeda; serves as a consultant for AbbVie, Celgene, Janssen, Pfizer, and Takeda; and has conducted clinical trials for AbbVie, Boehringer Ingelheim, Celgene Corp., Crohn's \& Colitis Foundation of America, Genentech, Gilead, Hollister, Medimmune, Pfizer, Receptos, Schwarz Pharma, Seres Therapeutics, and Takeda. His spouse serves on the Board of Directors for Aerpio Therapeutics, Novus Therapeutics, and Vital Therapies, Inc. Pekow J is a consultant for Versatem and 
CVS Caremark and has received grant support from AbbVie and Takeda. He has served on the advisory board for Takeda, Pfizer and Jansen. Dalal SR is on the speaker's bureau of AbbVie and has served as a consultant for Pfizer. The other authors declare no conflict of interest related to this work.

Rubin DT is an editorial board member of the journal but was not involved in the peer reviewer selection, evaluation, or decision process of this article. No other potential conflicts of interest relevant to this article were reported.

\section{Author Contribution}

Guarantor of article: Ayoub F. Conceptualization: Ayoub F, Odenwald M, Micic D, Dalal SR, Pekow J, Cohen RD, Rubin DT, Sakuraba A. Data curation: Ayoub F, Odenwald M. Formal analysis: Ayoub F. Investigation: Odenwald M, Dalal SR, Pekow J, Cohen RD, Rubin DT, Sakuraba A. Methodology: Ayoub F, Odenwald M. Project administration: Ayoub F. Software: Ayoub F. Supervision: Micic D, Dalal SR, Pekow J, Cohen RD, Rubin DT, Sakuraba A. Writing - original draft: Ayoub F. Writing - review \& editing: Ayoub F, Odenwald M, Micic D. Approval of final manuscript: all authors.

\section{ORCID}

Ayoub F

Odenwald M

Micic D

Dalal SR

Pekow J

Cohen RD

Rubin DT

Sakuraba A

https://orcid.org/0000-0001-8559-5477 https://orcid.org/0000-0003-1740-6472 https://orcid.org/0000-0003-2864-1670 https://orcid.org/0000-0003-1207-0500 https://orcid.org/0000-0002-4790-8994 https://orcid.org/0000-0002-6114-8936 https://orcid.org/0000-0001-5647-1723 https://orcid.org/0000-0003-2519-6129

\section{Supplementary Material}

Supplementary materials are available at the Intestinal Research website (https://www.irjournal.org).

\section{REFERENCES}

1. Siegmund B, Feakins RM, Barmias G, et al. Results of the Fifth Scientific Workshop of the ECCO (II): pathophysiology of perianal fistulizing disease. J Crohns Colitis 2016;10:377-386.

2. Schwartz DA, Loftus EV Jr, Tremaine WJ, et al. The natural history of fistulizing Crohn's disease in Olmsted County, Minnesota. Gastroenterology 2002;122:875-880.

3. Schwartz DA, Tagarro I, Carmen Díez M, Sandborn WJ. Prevalence of fistulizing Crohn's disease in the United States: esti- mate from a systematic literature review attempt and population-based database analysis. Inflamm Bowel Dis 2019;25: 1773-1779.

4. Present DH, Rutgeerts P, Targan S, et al. Infliximab for the treatment of fistulas in patients with Crohn's disease. N Engl J Med 1999;340:1398-1405.

5. Colombel JF, Sandborn WJ, Rutgeerts P, et al. Adalimumab for maintenance of clinical response and remission in patients with Crohn's disease: the CHARM trial. Gastroenterology 2007; 132:52-65.

6. Dewint P, Hansen BE, Verhey E, et al. Adalimumab combined with ciprofloxacin is superior to adalimumab monotherapy in perianal fistula closure in Crohn's disease: a randomised, double-blind, placebo controlled trial (ADAFI). Gut 2014;63: 292-299.

7. West RL, van der Woude CJ, Hansen BE, et al. Clinical and endosonographic effect of ciprofloxacin on the treatment of perianal fistulae in Crohn's disease with infliximab: a double-blind placebo-controlled study. Aliment Pharmacol Ther 2004;20: 1329-1336.

8. Sandborn WJ, Feagan BG, Rutgeerts P, et al. Vedolizumab as induction and maintenance therapy for Crohn's disease. $\mathrm{N}$ Engl J Med 2013;369:711-721.

9. Wang Y, Wang J, Pekow J, et al. Outcome of elective switching to vedolizumab in inflammatory bowel disease patients under tumor necrosis factor antagonist-maintained clinical remission. J Gastroenterol Hepatol 2019;34:2090-2095.

10. Page MJ, Shamseer L, Tricco AC. Registration of systematic reviews in PROSPERO: 30,000 records and counting. Syst Rev 2018;7:32.

11. Chapuis-Biron C, Bourrier A, Nachury M, et al. Vedolizumab for perianal Crohn's disease: a multicentre cohort study in 151 patients. Aliment Pharmacol Ther 2020;51:719-727.

12. Schwartz D, Peyrin-Biroulet L, Lasch K, Adsul S, Danese S. A randomized double-blind phase 4 study to evaluate the safety and proportion of subjects with fistula healing in 2 dose regimens of Entyvio (vedolizumab IV) in the treatment of fistulizing Crohn's disease (ENTERPRISE) [Internet]. c2020 [cited 2020 Jul 1].https://clinicaltrials.gov/ct2/show/NCT02630966.

13. Wells GA, Shea B, O'Connell D, et al. The Newcastle-Ottawa Scale (NOS) for assessing the quality of nonrandomised studies in meta-analyses [Internet]. c2000 [cited 2021 May 1]. http://www.ohri.ca/programs/clinical_epidemiology/oxford. asp.

14. Higgins JPT, Thomas J, Chandler J, et al. Cochrane handbook for systematic reviews of interventions. 2nd ed. Cochrane Col- 
laboration and John Wiley \& Sons Ltd., 2019.

15. Nyaga VN, Arbyn M, Aerts M. Metaprop: a Stata command to perform meta-analysis of binomial data. Arch Public Health 2014;72:39.

16. Feagan BG, Schwartz D, Danese S, et al. Efficacy of vedolizumab in fistulising Crohn's disease: exploratory analyses of data from GEMINI 2. J Crohns Colitis 2018;12:621-626.

17. Pestour S, Nancey S, Charlois A, et al. Influence of disease location on vedolizumab effectiveness in inflammatory bowel diseases: a real-life multicenter experience. Hum Health Pathol dumas-01623835 [Preprint]. 2017 [cited 2021 May 1]. https: //dumas.ccsd.cnrs.fr/dumas-01623835.

18. Schwartz DA, Peyrin-Biroulet L, Lasch K, Adsul S, Danese S. 949 Efficacy and safety of 2 vedolizumab iv regimens in patients with perianal fistulizing Crohn's disease: results of the ENTERPRISE study. Gastroenterology 2020;158(6 Suppl 1):S193-S-194.

19. Pestour S, Nancey S, Charlois AL, et al. DOP050 Influence of disease location on vedolizumab efficacy in inflammatory bowel disease: a real-life multicentre experience. J Crohns Colitis 2018;12(Suppl 1):S065-S066.

20. Schreiber S, Lawrance IC, Thomsen OØ, Hanauer SB, Bloomfield R, Sandborn WJ. Randomised clinical trial: certolizumab pegol for fistulas in Crohn's disease. Subgroup results from a placebo-controlled study. Aliment Pharmacol Ther 2011;33: 185-193.

21. Sands BE, Anderson FH, Bernstein CN, et al. Infliximab maintenance therapy for fistulizing Crohn's disease. N Engl J Med 2004;350:876-885.

22. Lee MJ, Parker CE, Taylor SR, et al. Efficacy of medical therapies for fistulizing Crohn's disease: systematic review and meta-analysis. Clin Gastroenterol Hepatol 2018;16:1879-1892.

23. Barré A, Colombel JF, Ungaro R. Review article: predictors of response to vedolizumab and ustekinumab in inflammatory bowel disease. Aliment Pharmacol Ther 2018;47:896-905.

24. Feagan B. Safety and positioning of vedolizumab in patients with inflammatory bowel disease. Gastroenterol Hepatol (N Y) 2018;14:244-246.

25. Wyant T, Leach T, Sankoh S, et al. Vedolizumab affects antibody responses to immunisation selectively in the gastrointestinal tract: randomised controlled trial results. Gut 2015; 64:77-83.
26. van Deventer SJ. Review article: targeting TNF alpha as a key cytokine in the inflammatory processes of Crohn's disease. The mechanisms of action of infliximab. Aliment Pharmacol Ther 1999;13 Suppl 4:3-8.

27. Panés J, Rimola J. Perianal fistulizing Crohn's disease: pathogenesis, diagnosis and therapy. Nat Rev Gastroenterol Hepatol 2017;14:652-664.

28. Scharl M, Rogler G. Pathophysiology of fistula formation in Crohn's disease. World J Gastrointest Pathophysiol 2014;5:205212.

29. Frei SM, Pesch T, Lang S, et al. A role for tumor necrosis factor and bacterial antigens in the pathogenesis of Crohn's diseaseassociated fistulae. Inflamm Bowel Dis 2013;19:2878-2887.

30. Scharl M, Weber A, Fürst A, et al. Potential role for SNAIL family transcription factors in the etiology of Crohn's disease-associated fistulae. Inflamm Bowel Dis 2011;17:1907-1916.

31. de Krijger M, Buskens CJ, Wildenberg ME, Verseijden C, de Jonge WJ, Ponsioen CY. P044 T cells expressing integrin $\alpha 4 \beta 7$ are abundant in fistula tracts of Crohn's disease patients. J Crohns Colitis 2018;12(Suppl 1):S114.

32. Hanauer SB, Sandborn WJ, Rutgeerts P, et al. Human anti-tumor necrosis factor monoclonal antibody (adalimumab) in Crohn's disease: the CLASSIC-I trial. Gastroenterology 2006; 130:323-333.

33. Sandborn WJ, Feagan BG, Radford-Smith G, et al. CDP571, a humanised monoclonal antibody to tumour necrosis factor alpha, for moderate to severe Crohn's disease: a randomised, double blind, placebo controlled trial. Gut 2004;53:1485-1493.

34. Sandborn WJ, Feagan BG, Stoinov S, et al. Certolizumab pegol for the treatment of Crohn's disease. N Engl J Med 2007;357: 228-238.

35. Sandborn WJ, Rutgeerts P, Enns R, et al. Adalimumab induction therapy for Crohn disease previously treated with infliximab: a randomized trial. Ann Intern Med 2007;146:829-838.

36. Harris MS, Wichary J, Zadnik M, Reinisch W. Competition for clinical trials in inflammatory bowel diseases. Gastroenterology 2019;157:1457-1461.

37. Lopez N, Ramamoorthy S, Sandborn WJ. Recent advances in the management of perianal fistulizing Crohn's disease: lessons for the clinic. Expert Rev Gastroenterol Hepatol 2019;13: $563-577$. 


\section{See "Vedolizumab for perianal fistulizing Crohn's disease: systematic review and meta-analysis" on pages $240-250$.}

Supplementary Table 1. Search Strategy, Search from Inception through June 2020

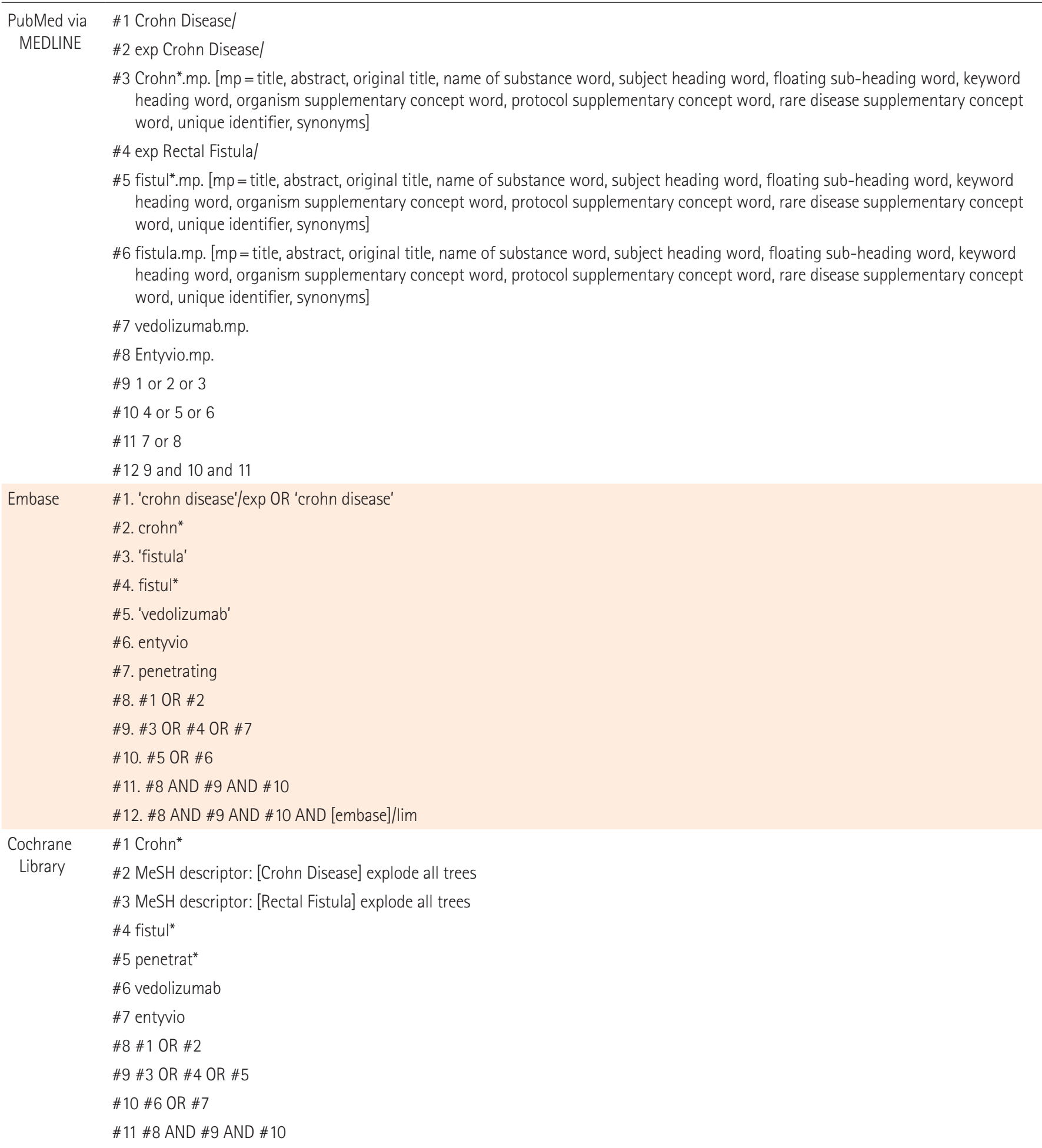


Supplementary Table 2. Modified Newcastle-Ottawa Quality Assessment Scale for Included Studies

\begin{tabular}{|c|c|c|c|c|c|c|c|}
\hline \multirow{2}{*}{ First author (year) } & \multicolumn{3}{|c|}{ Selection $^{\mathrm{a}}$} & \multicolumn{3}{|c|}{ Outcome $^{b}$} & \multirow{2}{*}{ Total score } \\
\hline & 1 & 2 & 3 & 1 & 2 & 3 & \\
\hline Pestour (2018) ${ }^{1}$ & - & + & + & - & + & + & 4 \\
\hline Feagan $(2018)^{2}$ & + & + & + & + & + & + & 6 \\
\hline Chapuis-Biron (2020) & + & + & + & + & + & + & 6 \\
\hline Schwartz $(2020)^{4}$ & - & + & + & + & + & + & 5 \\
\hline
\end{tabular}

a Selection: 1, representativeness of the exposed cohort (adequately describes baseline characteristics, previous/concomitant therapies of patients started on vedolizumab therapy); 2, ascertainment of exposure (adequately describes study definitions of perianal fistulizing disease adequately); 3 , outcome of interest not present at start of study.

${ }^{b}$ Outcome assessment: 1, assessment of outcome (adequately describes definition of perianal fistulizing disease healing); 2, adequacy of duration of follow-up; 3 , adequacy of completeness of follow-up (all subjects accounted for at end of study).

\section{REFERENCES}

1. Pestour S, Nancey S, Charlois A, et al. Influence of disease location on vedolizumab effectiveness in inflammatory bowel diseases: a real-life multicenter experience. Hum Health Pathol dumas-01623835 [Preprint]. 2017 [cited 2021 May 1].https://dumas.ccsd.cnrs.fr/ dumas-01623835.

2. Feagan BG, Schwartz D, Danese S, et al. Efficacy of vedolizumab in fistulising Crohn's disease: exploratory analyses of data from GEMINI 2. J Crohns Colitis 2018;12:621-626.

3. Chapuis-Biron C, Bourrier A, Nachury M, et al. Vedolizumab for perianal Crohn's disease: a multicentre cohort study in 151 patients. Aliment Pharmacol Ther 2020;51:719-727.

4. Schwartz D, Peyrin-Biroulet L, Lasch K, Adsul S, Danese S. A randomized double-blind phase 4 study to evaluate the safety and proportion of subjects with fistula healing in 2 dose regimens of Entyvio (vedolizumab IV) in the treatment of fistulizing Crohn's disease (ENTERPRISE) [Internet]. c2020 [cited 2020 Jul 1].https://clinicaltrials.gov/ct2/show/NCT02630966. 


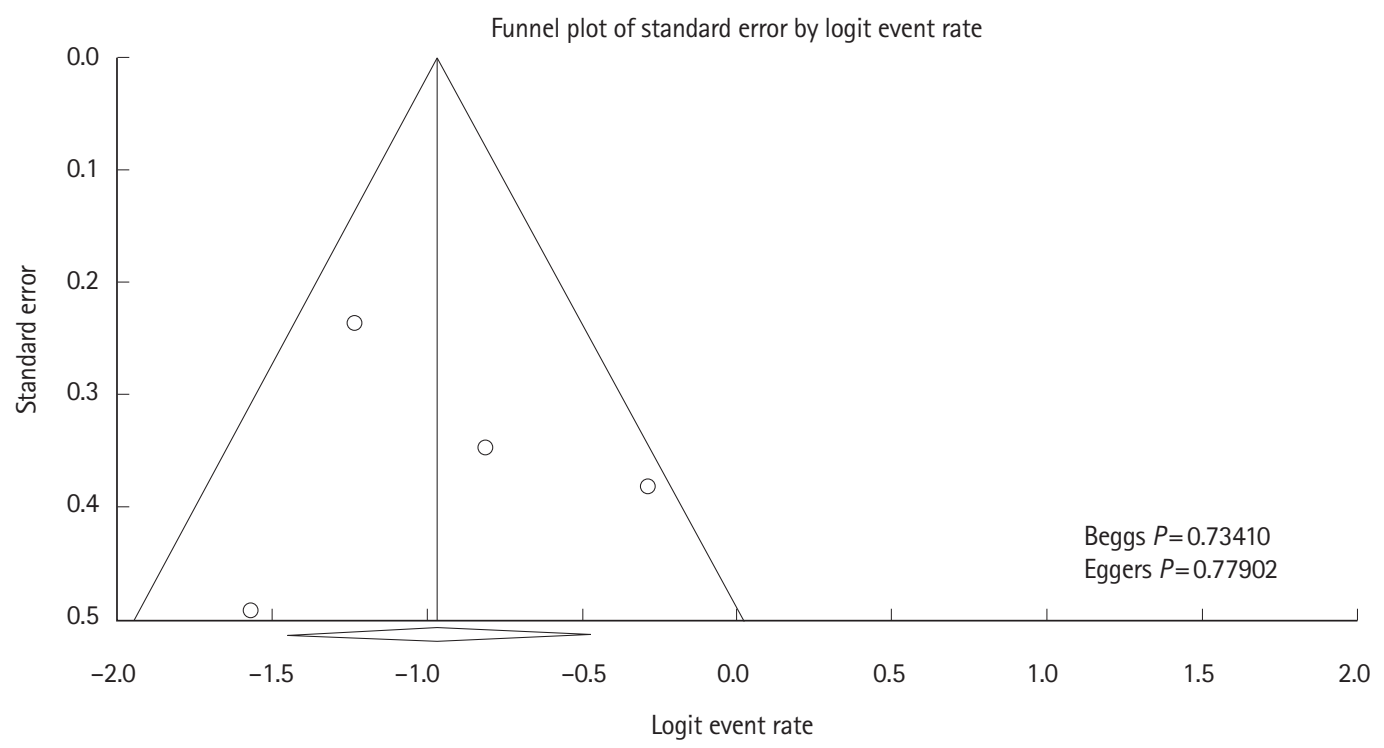

Supplementary Fig. 1. Funnel plot for included studies reporting complete healing of perianal fistulizing disease. 